\title{
Endovascular treatment of aortic arch aneurysm with a single-branched double-stage stent graft
}

\author{
Augusto D'Onofrio, MD, PhD, ${ }^{\mathrm{a}}$ Michele Antonello, MD, PhD, ${ }^{\mathrm{b}}$ Mario Lachat, $\mathrm{MD},{ }^{\mathrm{c}}$ David Planer, $\mathrm{MD},{ }^{\mathrm{d}}$ \\ Andrea Manfrin, BSc, ${ }^{\mathrm{e}}$ Andrea Bagno, BSc, ${ }^{\mathrm{e}}$ David Pakeliani, MD, ${ }^{\mathrm{c}}$ Franco Grego, MD, ${ }^{\mathrm{b}}$ and \\ Gino Gerosa, MD, ${ }^{\mathrm{a}}$ Padova, Italy; Zurich, Switzerland; and Jerusalem, Israel
}

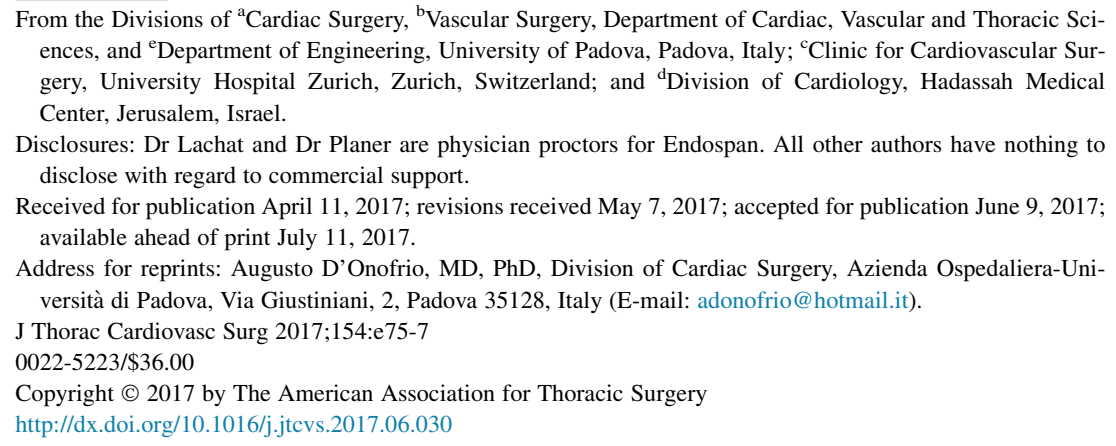
Video clip is available online.

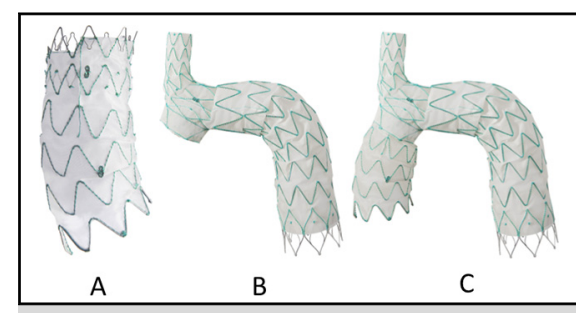

A, The ascending module, (B) the main module with its side branch for the brachiocephalic artery, and (C) the final assembled device.

\section{Central Message}

Endovascular stent grafting of the aortic arch is a new promising emerging technology that allows the exclusion of aortic arch aneurysms through a micro-invasive procedure.

See Editorial Commentary page e79.
Stent grafting for the treatment of aortic arch pathologies is the new frontier of endovascular aortic surgery because it allows the exclusion of aortic arch aneurysm through a microinvasive procedure $^{1,2}$ with no cardiopulmonary bypass, aortic crossclamping, and circulatory arrest, which commonly are needed during conventional surgery. We hereby describe for the first time the use of a new endovascular system, Nexus (Endospan, Herzlia, Israel), for the treatment of aortic arch lesions. Nexus is made of 2 components: (1) a main module, for the aortic arch and the descending aorta with a side-branch for the brachiocephalic artery (BCA) and (2) a curved module for the ascending aorta that connects to the main module through a side-facing self-protecting sleeve and lands into the sinotubular junction (Figure 1). Before Nexus positioning, an extraanatomic surgical debranching of supra-aortic vessels, with the use of the BCA as the main source of blood, is required to provide adequate cerebral blood flow.

\section{CASE REPORT}

A 74-year-old patient was referred to our center with diagnosis of aortic arch aneurysm. The preoperative computed tomography (CT) reconstruction showed a 6.5$\mathrm{cm} \times 6-\mathrm{cm}$ aneurysm on the anterior portion of the aortic arch (Figure 2, A). Surgery was indicated and proposed to the patient but, since he was a Jehovah's witness, he denied it because of the high risk of blood transfusions. Therefore, hybrid correction of the aortic arch aneurysm was planned.

The patient underwent extra-anatomic supra-aortic reconstruction with right carotid to left subclavian bypass (both end-to-side) with an 8-mm polytetrafluoroethylene vascular graft and end-to-side reimplantation of the right carotid artery on the graft. After 1 week, the endovascular stent-grafting procedure was performed. Under general anesthesia and with bilateral percutaneous femoral access, the main module was introduced via right brachiofemoral through-and-through guidewire landing with the sidebranch in the BCA and covering the arch and the descending aorta. Subsequently, via a guidewire placed in the left ventricle and during rapid pacing the ascending aorta module was implanted. taking care of positioning the " $\mathrm{B}$ " shaped radiopaque marker of the ascending module at the level of the tantalum ring of the main module in order to achieve perfect sealing and to avoid coverage of the BCA branch.

Figure 2, $B$ shows the final result with complete exclusion of the aneurysm, good sealing between the 2 components, and good perfusion of all supra-aortic vessels with blood flow originating from the stented BCA. Video 1 shows the procedural steps of Nexus implantation. Total procedural time was 120 minutes, overall fluoroscopy time was 


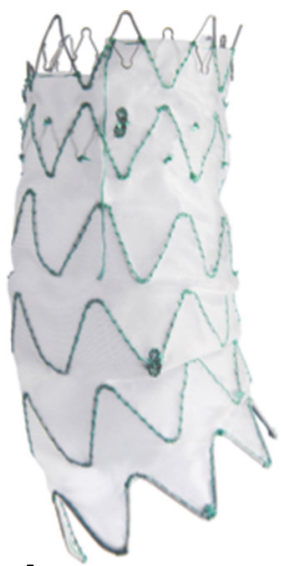

A

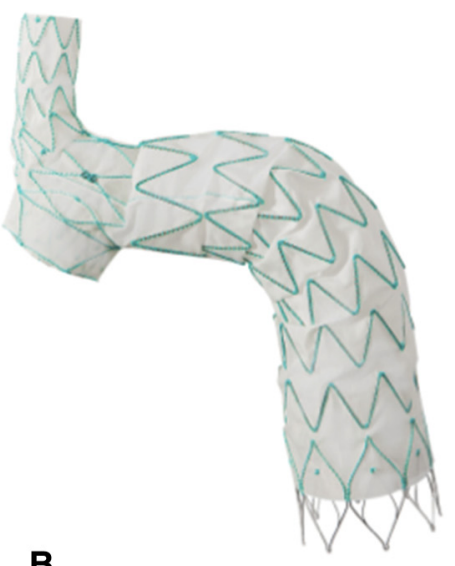

B

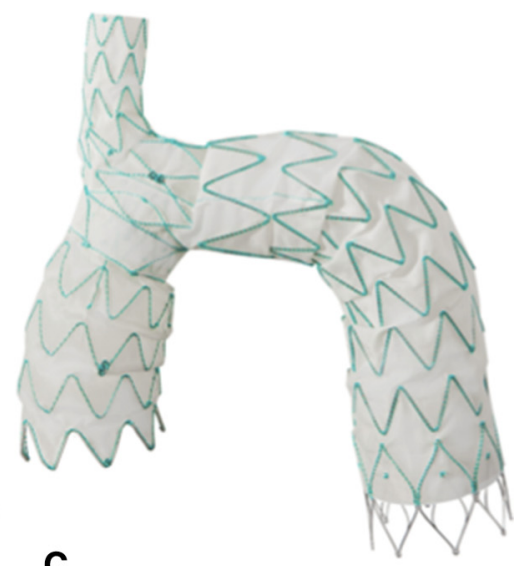

C

FIGURE 1. A, The ascending module, (B) the main module with its side branch for the brachiocephalic artery, and (C) the final assembled device.

36 minutes, and contrast volume was $140 \mathrm{~mL}$. A few days later, the patient underwent percutaneous closure of the ostium of the left subclavian artery with a 14-French vascular plug. The patient, immediately after Nexus implantation, experienced transient paresis of the right hand, brain CT scan was normal, and he completely recovered a few days later. The patient was discharged home in good clinical condition; no transfusions were required during hospital stay. At 6-month follow-up the patient was in good clinical conditions, and the CT scan showed stable results with complete exclusion of the aneurysm and no endoleaks.
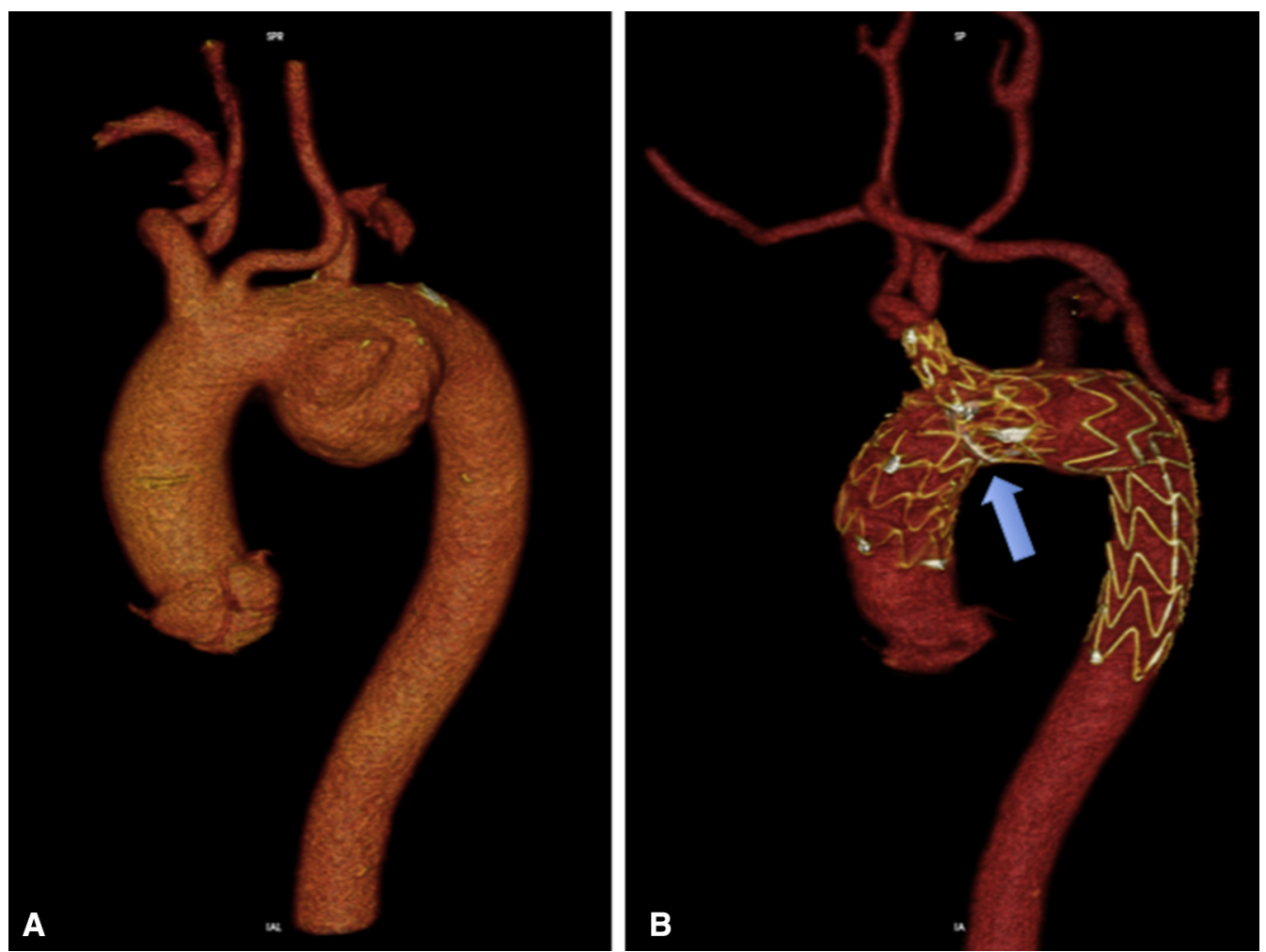

FIGURE 2. A, Preoperative angio-computed tomography 3-dimensional reconstruction of the aortic arch aneurysm. B, Postoperative angio-computed tomography reconstruction. The arrow shows the anastomotic area (tantalum ring) between the 2 modules. Patency of the supra-aortic debranching that provides adequate brain perfusion is also evident. 


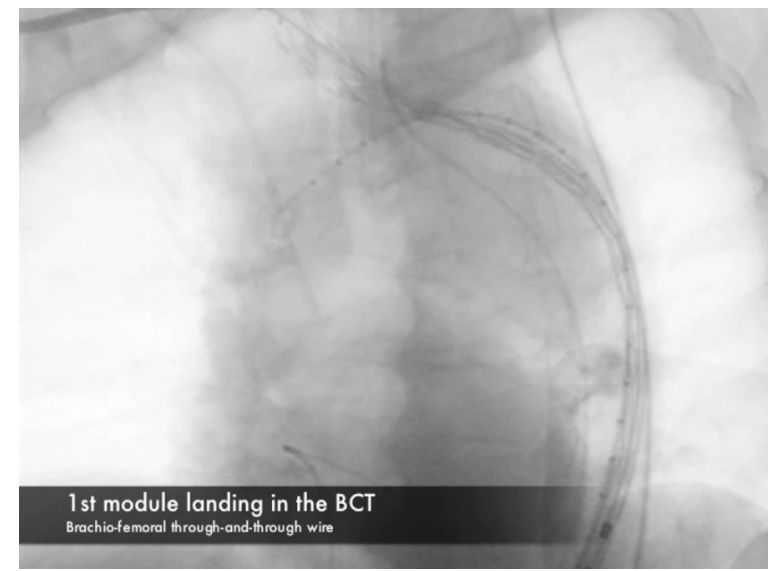

VIDEO 1. The steps of Nexus endograft implantation. The distal portion of the first module lands in the brachiocephalic trunk (BCT) and then stent opening continues into the aortic arch and the descending aorta. In this particular case, the system is pushed forward during the opening of the side-facing self-protecting sleeve to optimize its position toward the ascending aorta. The landing zone for the second module is identified easily by a tantalum radio-opaque ring. Then, postdilatation of the origin of the BCT is performed. The second module is advanced in the ascending aorta on a guidewire positioned in the left ventricle, as for a transfemoral aortic valve implantation procedure. The second module is deployed during rapid pacing. Rapid pacing should be as short as possible but long enough to ensure adequate, stable, and safe opening of the ascending module in its proper position. We believe that with experience pacing run duration can be as short as that performed in a transcatheter aortic valve implantation procedure. Balloon postdilatation of the connection zone between the 2 modules also is performed during rapid pacing. The final angiography shows a good positioning of the stent graft, good sealing between the 2 components, and good perfusion of all supra-aortic vessels. Video available at: http://www.jtcvsonline.org/article/S0022-5223(17)31240-0/ addons.

\section{DISCUSSION}

This paper describes for the first time the implantation of Nexus Endospan branched aortic arch stent graft. Surgery for aortic arch pathologies such as aneurysms and chronic dissections requires invasive procedures that need cardiopulmonary bypass, aortic crossclamp, hypothermic circulatory arrest, and selective cerebral perfusion. The endovascular microinvasive treatment of these diseases could be an interesting option, especially in patients deemed at high risk for surgery. There are already reports of endovascular stenting of the aortic arch..$^{2-4}$ In particular, Patel and colleagues ${ }^{5}$ described the results of the Gore Thoracic Branch Endoprosthesis (TBE) in the treatment of distal aortic arch and descending thoracic aneurysms. This device, similarly to Nexus, has a side branch that may be positioned in one of the supra-aortic vessels.

However, there are several differences between these 2 devices. Nexus has 2 modules that allow landing in zone 0 with a complete exclusion of the ascending aorta, the aortic arch, and the descending aorta. In fact, Nexus proximal landing may be at the sinotubular junction level, thus enabling the treatment of arch aneurysms that also involve the ascending aorta. Furthermore, the side branch of the Nexus is incorporated in the main module, whereas in the Gore device, it has to be implanted separately. In conclusion, aortic arch stent-graft with Endospan Nexus is a new promising technique for the endovascular treatment of aortic arch pathologies, potentially having long-term migration resistance because of its landing in the BCA. A nextgeneration configuration, incorporating a precannulated fenestration for the left common carotid artery, could avoid the supra-aortic surgical debranching procedure.

\section{References}

1. D'Onofrio A, Gerosa G. Shifting a paradigm of cardiac surgery: from minimally invasive to micro-invasive. J Heart Valve Dis. 2015;24:528-30.

2. Joseph G, Canaud L. Combining ascending aorta and aortic arch TEVAR. J Endovasc Ther. 2017;24:81-3.

3. Kölbel T, Detter C, Carpenter SW. Acute type A aortic dissection treated using a tubular stent-graft in the ascending aorta and a multibranched stent-graft in the aortic arch. J Endovasc Ther. 2017;24:75-80.

4. Bernardes RC, Navarro TP, Reis FR, Lima LC, Monteiro EL, Procopio RJ, et al Early experience with off-the-shelf endografts using a zone 0 proximal landing site to treat the ascending aorta and arch. J Thorac Cardiovasc Surg. 2014;148:105-12.

5. Patel HJ, Dake MD, Bavaria JE, Singh MJ, Filinger M, Fischbein MP, et al. Branched endovascular therapy of the distal aortic arch: preliminary results of the feasibility multicenter trial of the gore thoracic branch endoprosthesis. Ann Thorac Surg. 2016;102:1190-8. 